\title{
TERAPIA DE ACEPTACIÓN Y COMPROMISO: DESCRIPCIÓN GENERAL DE UNA APROXIMACIÓN CON ÉNFASIS EN LOS VALORES PERSONALES
}

\section{ACCEPTANCE AND COMMITMENT THERAPY: OVERVIEW OF A CLINICAL APPROACH WITH EMPHASIS ON PERSONAL VALUES}

\author{
Luis Francisco Vargas Madriz* \\ Ronald Ramírez Henderson **
}

RESUMEN

Este artículo hace un recuento de la Terapia de Aceptación y Compromiso (ACT), la cual es una aproximación clínica conductual que toma como referencia los valores de la persona y su experiencia para guiar el proceso de intervención psicoterapéutica. El artículo describe sus fundamentos filosóficos y teóricos, las premisas de la terapia, los objetivos generales, los métodos empleados en el trabajo clínico y los principales componentes del tratamiento. Se finaliza con un análisis crítico de la incursión de la ACT en Costa Rica.

PALABRAS CLAVE: PSICOLOGÍA * TERAPIA * ASPECTOS PSICOLÓGICOS * SALUD MENTAL * COMPORTAMIENTO

\section{ABSTRACT}

This article reviews the Acceptance and Commitment Therapy (ACT), which is a behavioral clinical approach with the patient's personal values and personal experiences as reference points to the psychotherapeutic intervention. The article describes the philosophical and theoretical foundations, premises of the therapy, general objectives, methods of clinical work, and main components of treatment. It finalizes with a critical analysis the implementation of ACT in Costa Rica.

KEYWORDS: PSYCHOLOGY * THERAPY * PSYCHOLOGICAL ASPECTS * MENTAL HEALTH * BEHAVIOR

\footnotetext{
* Unidad de Investigación de la Fundación Omar Dengo y Escuela de Psicología de la Universidad de Costa Rica (UCR). luis.vargasmadriz@ucr.ac.cr

** Escuela de Psicología de la Universidad de Costa Rica (UCR). ronald.ramirez@ucr.ac.cr
} 


\section{INTRODUCCIÓN}

La Terapia de Aceptación y Compromiso (ACT, Acceptance and Commitment Therapy), surge como una aproximación clínica conductual basada en los valores personales de la persona consultante más que como un modelo de tratamiento con fases de intervención definidas (Páez, Gutiérrez, Valdivia y Luciano, 2006; Robb, 2007; Wilson y Luciano, 2002). La ACT parte de los postulados de la teoría del marco relacional (Hayes, Barnes-Homes y Roche, 2001), como una forma de intervención psicoterapéutica conductual que pone especial énfasis sobre el influjo de la evitación experiencial en los distintos problemas que presentan los consultantes (Gaudiano, 2009; Hofmann, 2008; Rey, 2004; Ruiz y Luciano, 2009; Wilson y Luciano, 2002; Yovel, 2009).

Las características particulares de este tipo de intervención hacen que sea una de las propuestas más flexibles de tratamiento en la actualidad. La ACT ha sido puesta en práctica en una variedad de poblaciones, diversidad de problemáticas e igualmente en múltiples características de los terapeutas. Asimismo, ha sido empleada con variaciones en la duración de la intervención, desde aplicaciones extensas de varios meses hasta intervenciones breves (Wilson y Luciano, 2002).

La ACT es una postura del conductismo radical (Baum, 1994; Skinner, 1953, 1969, 1974), que combina estrategias de aceptación $y$ toma de consciencia del comportamiento propio por parte de los individuos (Strosahl y Robinson, 2008) como una forma de consecución del cambio conductual, por lo que la puesta en práctica de dichas destrezas va a influir directamente en la mejora de la "flexibilidad psicológica" de los consultantes (Arch y Craske, 2008; Berman, Boutelle y Crow, 2009; Carrascoso y Valdivia, 2009; Ossman, Wilson, Storaasli y McNeill, 2006).

La flexibilidad psicológica constituye la habilidad de la persona para entrar en contacto con el presente de una forma más completa como ser humano consciente y a partir de dicho contacto, se decide cambiar el comportamiento problemático o persistir en el comportamiento que se considera necesario para alcanzar metas (Gaudiano, 2009; Ossman, et ál., 2006; Twohig, et ál., 2010; Yovel, 2009).

\section{TERAPIA DE ACEPTACIÓN Y COMPROMISO}

La ACT es una terapia basada filosóficamente en el contextualismo funcional y teóricamente en la teoría del marco relacional (Gaudiano, 2009; Hayes et ál., 2001; Hofmann, 2008; Rey, 2004; Ruiz y Luciano, 2009; Vilardaga, Hayes y Schelin, 2007; Wilson y Luciano, 2002). El funcionalismo contextual es una filosofía pragmática desarrollada para clarificar ciertos problemas filosóficos que parecían permanecer inciertos en el análisis conductual y más concretamente, en el conductismo radical (Ruiz, 2010).

El contextualismo se identifica por caracterizar los eventos como acciones en curso a partir de un contexto determinado, por lo que las acciones se consideran eventos enteros que solamente pueden separarse para propósitos pragmáticos (Hayes, 2008; Hofmann, 2008; Ruiz, 2010; Vilardaga et ál., 2007; Yovel, 2009). El contexto se convierte en el punto a partir del cual, se trata de buscar una explicación del comportamiento humano, tomando en consideración que este va a estar determinado por las contingencias ambientales de un momento en particular, así como, la historia de aprendizaje que la persona haya tenido hasta dicho momento.

El contextualismo funcional es una variedad específica del contextualismo, cuya premisa básica es la predicción y la influencia de eventos con un amplio nivel de precisión, alcance $y$ profundidad. Debido a que el contextualismo funcional cuenta con estas metas, se trata de entender que cada elemento conductual tiene que ser explicado en términos de variables contextuales, de lo contrario, el comportamiento no podría ser influenciado eficientemente. Así, desde esta perspectiva, ni los pensamientos ni los sentimientos tienen una relación de causalidad directa con el comportamiento del sujeto (Hayes, 2008; Hofmann, 2008; Ruiz, 2010; Vilardaga et ál., 2007). Tanto los pensamientos como los sentimientos son interpretados como conductas al igual que el comportamiento motor; de esta forma 
se facilita la comprensión de las interacciones conducta-conducta al interior de la persona como sujeto (Wilson y Luciano, 2002).

La teoría del marco relacional es un acercamiento conductual contextual al lenguaje humano y a la cognición que actualmente cuenta con una amplia evidencia empírica de sus fundamentos. Dicha teoría se encuentra sustentada en las leyes que se establecieron con el análisis funcional de la conducta (Skinner, 1953, 1969 y 1974), pero representa un cambio cualitativo debido a que integra áreas dispares de la investigación conductual, cuyo supuesto central consiste en que los seres humanos aprenden a relacionar estímulos bajo controles contextuales arbitrarios (Hayes et ál., 2001; Hofmann, 2008; Ruiz, 2010; Yovel, 2009).

Esta aproximación tiene un amplio número de implicaciones dentro del área de psicoterapia, por ejemplo: los intentos directos de cambiar o eliminar el contenido de la red relacional o cambios de primer orden (Watzlawick, Weakland y Fisch, 1974), no están bien fundamentados psicológicamente debido a que conducen a una extensión arbitraria de la red y más bien, debido a que la respuesta relacional está controlada contextualmente (Ruiz, 2010), es posible cambiar directamente el contexto de las redes relacionales sin cambiar su contenido o cambio de segundo orden (Watzlawick et ál., 1974). De esta forma, se plantea que:

Al establecer la postura de aceptación psicológica, los eventos que anteriormente eran inherentemente problemáticos, se convierten en oportunidades para el crecimiento, interés, o comprensión. En otras palabras, la atención cambia de problemas con contenido histórico a problemas del funcionamiento actual (Hayes, 1994: 13).

La propuesta es un cimiento importante debido a que enfatiza la posibilidad de cambio a partir de la modificación de las contingencias ambientales (tanto externas o públicas, como internas o privadas) que se encuentran controlando la conducta del individuo. Así, dentro de la propuesta de la ACT, la teoría del marco relacional juega un importante papel debido a las particularidades del trastorno del cual se fundamenta. A partir de estos postulados, la ACT brinda una conceptualización del Trastorno de Evitación Experiencial (TEE), el cual parte de la premisa que el sufrimiento psicológico “(...) es parte inseparable de la vida humana en tanto que ésta incluye circunstancias y comunidades que conforman contextos verbales a través de los cuales una persona determinada reaccionará verbalmente a numerosos eventos, incluida su propia conducta" (Wilson y Luciano, 2002: 73). Los autores mencionan que estos contextos representan las condiciones necesarias para evaluar los eventos externos, así como, el propio comportamiento, como reacciones normales (aún cuando causen dolor a las personas) o como reacciones anormales y negativas.

El TEE se caracteriza por la ocurrencia de esfuerzos deliberados de evitar y/o escapar de eventos privados como emociones, pensamientos, recuerdos $y$ sensaciones corporales que son vividas por la persona como aversivas. Este comportamiento es reforzado negativamente por la evitación o disminución del sentimiento de incomodidad vivido (Blackledge y Hayes, 2001; Gaudiano, 2009; Ruiz, 2010; Ruiz y Luciano, 2009; Wilson y Luciano, 2002; Yovel, 2009). El trastorno presenta dos características principales:

1. El TEE es de naturaleza verbal. Debido a que la regulación es verbal y se encuentra presente cuando el individuo no se encuentra dispuesto a entrar en contacto con sus experiencias privadas vividas de forma aversiva, por lo que termina comportándose de forma deliberada para alterar dichas experiencias, así como, las condiciones que las generan (Hayes et ál., 1996 citados en Wilson y Luciano, 2002).

2. El TEE es una clase funcional limitante, por lo que el individuo que lo experimenta ha aprendido a responder a los eventos privados vividos de forma aversiva con la consecuente eliminación o evitación de dicho evento privado en una variedad de reacciones que dependen de la historia de cada individuo (Wilson y Luciano, 2002). 
Es necesario aclarar que la evitación experiencial no es un problema psicológico en sí mismo, pero se convierte en una dificultad cuando imposibilita a la persona a realizar de forma adecuada sus diversas labores cotidianas, lo que dentro del marco de la АCT se conceptualiza como inflexibilidad psicológica (Blackledge y Hayes, 2001; Gaudiano, 2009; Ruiz, 2010; Ruiz y Luciano, 2009; Wilson y Luciano, 2002; Yovel, 2009).

\section{PREMISAS DE LA TERAPIA DE ACEPTACIÓN Y COMPROMISO}

La ACT enfatiza el actuar como la premisa básica para una orientación centrada en los valores del individuo. Wilson y Luciano plantean que la ACT es "(...) un modelo de salud y no de enfermedad, ya que se entiende que el sufrimiento es universal y su causa principal es la información del lenguaje en áreas en las que no es funcional o no es útil" (2002: 97).

El proceso terapéutico en la ACT tiene como premisa fundamental que la experiencia de dolor psicológico es inevitable y que la utilización de la evitación experiencial y demás mecanismos de afrontamiento similares para lidiar con el malestar, usualmente incrementan el sufrimiento en las personas aunque sea comprensible su empleo (Wilson y Luciano, 2002; Yovel, 2009). Entonces, la propuesta del modelo implica la aceptación de los eventos privados propios como un compromiso optado con la vida $y$ por ende, un compromiso con cualesquiera eventos privados que, dependiendo de la historia del sujeto, se presenten (Wilson y Luciano, 2002).

La persona cuando inicia el proceso de intervención psicoterapéutica se encuentra atrapada en el patrón de evitación, en una suerte de lucha inquebrantable por dejar de estar en dicho patrón. Este esquema se muestra como un callejón sin salida, debido a que la lucha por eliminar o reducir el malestar hace que las acciones llevadas a cabo dirijan a la persona a otro estado de malestar continuo, por lo que la persona siempre se encuentra experimentando sufrimiento psicológico (Wilson y Luciano, 2002).

La ACT ejerce influencia directa sobre los contextos verbales en los que se encuentran las personas consultantes, por lo que se procura que la persona reflexione en torno a los eventos privados como lo que realmente son, tratando de elegir una nueva perspectiva de vida que incluya la aceptación de dichos eventos y proponerse el seguir dicha dirección. En este contexto, la aceptación “(...) implicaría el no tratar de evitar, controlar, o cambiar cualquier elemento de las contingencias relevantes que operan en un momento dado" (Dougher, 1994: 38).

De esta forma, Wilson y Luciano (2002), mencionan que esta perspectiva considera:

$\diamond \quad$ El tratamiento centrado en los valores del sujeto como vehículo para el cambio conductual.

$\diamond \quad$ Da relevancia de la experiencia que la persona aporta al proceso de intervención terapéutica.

$\diamond \quad$ La experiencia actual de la persona consultante va a indicar que actuar evitando el malestar experimentado, conduce a problemáticas dentro de la propia vivencia. Usualmente la persona queda atrapada en lo que cree es la solución a sus problemas, cuando en realidad eso es parte del problema real.

$\diamond \quad$ La experiencia terapéutica en ACT va a promover una nueva dirección de trabajo en donde se orienta a centrarse en los caminos valiosos para el individuo, a través del distanciamiento psicológico de los eventos temidos.

La ACT destaca claramente la experiencia subjetiva del individuo como principal insumo para la puesta en marcha del proceso psicoterapéutico. Es trascendental resaltar que la perspectiva parte de una valoración de las vivencias personales $y$ un profundo respeto por las estrategias de afrontamiento que se han puesto en práctica hasta el momento, ya que la intervención parte de estos datos para proponer una experiencia más completa y sana por parte del sujeto. Es la condición actual del sujeto la que por sí misma va a brindar la clave para la promoción del cambio conductual deseado en la persona consultante. Así, de acuerdo con Wilson y Luciano (2002), las premisas de la ACT son: 
1. Se asume que si la persona se comporta de acuerdo a sus propios valores, posteriormente va a sentirse mejor.

2. Se asume que el sufrimiento psicológico es normal en los seres humanos, siendo este una manifestación de que la persona se encuentra cerca de lo que realmente le importa.

3. Se asume que hay más vida en los momentos en que se experimenta dolor que en aquellos que se experimenta alegría.

4. Se asume que todas las personas, de una $\mathrm{u}$ otra manera, esperan vivir de forma más plena sus vidas.

\section{ESTRATEGIAS DE INTERVENCIÓN}

Los valores dentro de la ACT tienen un papel preponderante en el proceso de cambio de las personas (Dougher, 1994; Hayes, 1994; Páez et ál., 2006; Robb, 2007; Strosahl y Robinson, 2008; Wilson y Luciano, 2002). La propuesta de esta terapia se basa en la clarificación que la persona haga de sus propios valores, para que así aprenda a tomar decisiones más acertadas en torno a la dirección que debe tomar su vida. Lo importante es que el individuo pueda aprender a reconocer qué aspectos pueden ser cambiados y aquellos que definitivamente no puede cambiar. A partir de los fundamentos teóricos de la ACT este proceso se va a alcanzar a través de la modificación de los contextos verbales en los que los sujetos se encuentran atrapados (Hayes, 2008; Hofmann, 2008; Ruiz, 2010; Wilson y Luciano, 2002; Yovel, 2009).

Wilson y Luciano (2002), indican que los objetivos de la ACT son:

1. La clarificación de los valores de la persona consultante.

2. La aceptación de los eventos privados de la persona consultante, principalmente relacionados con aquellos acontecimientos que no pueden modificarse y que consecuentemente va a implicar el abandono de conductas de afrontamientos no efectivos y el saber escoger flexiblemente el comportamiento funcional y efectivo.
3. El fortalecimiento del "yo como contexto" para que la persona consultante sea capaz de contemplar sus eventos privados y a partir de la reflexión, elegir el camino más adecuado para sus propios intereses.

Como se puede observar, los objetivos de la intervención se encuentran focalizados en la propiciación del cambio a través de la aceptación de las particularidades propias del individuo, tomando una actitud crítica ante aquellos elementos de la experiencia subjetiva que no pueden ser modificados $y$ haciendo esfuerzos por adoptar estrategias de afrontamiento más constructivas para aquellos factores que tienen amplias probabilidades de cambio.

La consecución de dichos objetivos implica la puesta en práctica de ciertos métodos por parte del terapeuta, principalmente centrados en estilos de su propio discurso que se ponen en práctica para cambiar los patrones del contexto verbal, los cuales han atrapado al sujeto en los problemas que experimenta en la actualidad. Los métodos de este tipo son las metáforas, las paradojas y los ejercicios experienciales.

Las "metáforas" son un recurso valioso dentro de la ACT debido a que se establece un nuevo contexto verbal que valida el conocimiento del propio sujeto para quebrantar la lógica detrás del comportamiento de evitación. Por supuesto, las metáforas van a adoptar formas diversas dependiendo del contexto al que se haga referencia dentro de la sesión terapéutica. Asimismo, las "paradojas" son formulaciones que resaltan las contradicciones entre las creencias literales del individuo y las propiedades funcionales de las reglas de evitación (Wilson y Luciano, 2002).

La implementación de este tipo de métodos ayuda en el establecimiento de contextos verbales disímiles a los mantenidos hasta el momento por la persona consultante, por lo que se trata de acercamientos más directos a la problemática del individuo. Empero, también pueden ponerse en práctica "ejercicios experienciales" diseñados específicamente para que la persona establezca un contacto directo con 
los procesos verbales que hasta el momento ha estado evitando, de forma que pueda enfrentarse a las situaciones aversivas de su vivencia (Wilson y Luciano, 2002).

El "yo como contexto", por su parte, hace referencia al impulso que brinda el terapeuta a la persona para que adopte un sentimiento desde sí misma como locus de perspectiva, a través de la cual pueda experimentar una faceta más trascendental y espiritual del comportamiento humano (Blackledge y Hayes, 2001; Hofmann, 2008; Ruiz, 2010; Strosahl y Robinson, 2008; Yovel, 2009). Este método más directo, igualmente, le permite al terapeuta inducir un cambio positivo en el comportamiento del individuo.

Asimismo, dentro de este tipo de métodos también se encuentra la "defusión cognitiva" dónde se alienta a los individuos que reconozcan que cualquier intento por controlar eventos privados se convierte en uno de los principales problemas más que en la solución de los mismos (Blackledge y Hayes, 2001; Hofmann, 2008; Ruiz, 2010; Yovel, 2009).

\section{COMPONENTES DE LA TERAPIA DE ACEPTACIÓN Y COMPROMISO}

Tal y como se ha planteado, la ACT se sustenta en premisas que resaltan la importancia de los valores, que reconocen la normalidad del sufrimiento psicológico dentro de la vivencia humana y que existe una búsqueda dentro de todas las personas para conseguir un estado de experiencia más satisfactorio dentro de sus cotidianidades. Así, los componentes de la ACT son los valores, la exposición a los contextos aversivos, la desactivación de funciones y distanciamiento, $y$ el fortalecimiento de la persona consultante.

Los "valores" deben ser clarificados para que la persona pueda dirigirse en forma consciente y comprometida hacia un camino en que se puedan hacer modificaciones a la experiencia vivida hasta el momento. Es importante que la persona se pregunte si lo que ha hecho hasta el momento y que probablemente hace en la actualidad, ayuda a mejorar su experiencia vital en la dirección deseada, así como, si donde se encuentra es el lugar donde quiere estar en el futuro (Páez et ál., 2006; Robb, 2007; Strosahl y Robinson, 2008; Wilson y Luciano, 2002).

La "exposición" parte de la conceptualización de que las barreras existentes entre el lugar en donde la persona se encuentra y en el cual la persona quiere estar, son causadas por el patrón de evitación mantenido. Este componente permite que la persona consultante tenga contacto con dichas barreras, las cuales en realidad son eventos privados temidos, por lo que se sientan las bases para la desactivación del contexto verbal presentado (Dougher, 1994; Ruiz, 2010; Wilson y Luciano, 2002).

La "desactivación de las funciones" y el "distanciamiento" se encuentran sustentados en la postura del "yo como contexto", donde se trata de interrumpir las barreras creadas por las personas a través del establecimiento del contexto verbal, por lo que se procura reducir el dominio literal del lenguaje (Wilson y Luciano, 2002; Yovel, 2009).

Finalmente, es importante el "fortalecimiento de la persona" debido a que los valores se toman como un aspecto fundamental de trabajo dentro de la sesión de terapia. El proceso va a fortalecer a la persona consultante debido a que la dirección de trabajo va a ser establecida por sus vivencias particulares, por lo que siempre el individuo va a ser determinante en la decisión de la dirección que se va a tomar (Wilson y Luciano, 2002).

\section{TERAPIA DE ACEPTACIÓN Y COMPROMISO EN DISTINTAS POBLACIONES}

La ACT se ha mostrado especialmente efectiva en poblaciones que se encuentran luchando contra sus eventos privados de forma que han desarrollado TEE (Arch y Craske, 2008; Berman et ál., 2009; Blackledge y Hayes, 2001; Carrascoso y Valdivia, 2009; Gaudiano, 2009; Hofmann, 2008; Ruiz, 2010; Wilson y Luciano, 2002; Yovel, 2009). Empero, la propuesta psicoterapéutica también se ha mostrado efectiva en una serie de problemáticas distintas.

Existe evidencia de la efectividad de la ACT en población con problemas relacionados con la conducta alimentaria, en donde las participantes después de un proceso de intervención de cinco meses presentaron mejoras en 
las condiciones de salud en relación a esta problemática, así como en el seguimiento realizado al año de la intervención (Berman et ál., 2009).

Los problemas relacionados con la conducta de fumado también han probado tener un adecuado tratamiento con la ACT en comparación a la terapia cognitiva-conductual tradicional (Hernández-López, Luciano, Bricker, Roales-Nieto y Montesinos, 2009), incluso en contextos de tratamiento telefónico (Bricker, Mann, Marek, Liu y Peterson, 2010). Además, los problemas de consumo de otras sustancias adictivas también han sido abordados de forma positiva con la ACT, entre los que resalta el consumo de sustancias como estrategia de evitación experiencial (Luciano, Páez y Valdivia, 2010), el uso de anfetaminas (Smout et ál., 2010) y el consumo de alcohol (Petersen y Zettke, 2009).

La ACT también se ha mostrado efectiva en el tratamiento de problemas psicológicos diversos como la fobia social (Ossman et ál., 2006), el trastorno obsesivo compulsivo (Twohig et ál., 2010), el trastorno de pánico (Carrascoso y Valdivia, 2009) e incluso problemas de satisfacción sexual en parejas (Honarparvaran, Tabrizy, Navabinejad, Shafiabady y Moradi, 2010).

Finalmente, la ACT también ha mostrado buenos resultados en labores de mejoramiento del rendimiento en ajedrez en profesionales, presentándose mejoras significativas en los resultados obtenidos en competiciones posteriores al tratamiento (Ruiz y Luciano, 2009).

\section{DISCUSIÓN: TERAPIA DE ACEPTACIÓN \\ Y COMPROMISO EN EL CONTEXTO COSTARRICENSE}

La ACT se muestra en definitiva como una aproximación clínica apropiada para la intervención psicoterapéutica en el país. Empero, debido a sus fundamentos específicos dentro de la perspectiva del Conductismo Radical (Baum, 1994; Skinner, 1953, 1957, 1969 y 1974), es imprescindible que la "preparación de los terapeutas" tenga una adecuada comprensión de las bases epistemológicas de esta perspectiva psicológica, en miras de poner en práctica una aplicación ética del enfoque.
La ACT siendo una aproximación, más que un procedimiento psicoterapéutico estructurado, hace que su incorporación dentro de los diferentes sistemas de atención psicológica sea más sencilla. De esta manera, no es inverosímil propiciar la implementación de este tipo de estrategias dentro del contexto de atención pública brindada en el sistema de seguridad social del país y mucho menos dentro de la práctica privada de los profesionales de la salud mental costarricense.

Los "recursos necesarios" para la ejecución de la ACT no son en ninguna medida distintos de aquellos requeridos para la puesta en marcha de cualquier otra perspectiva de intervención psicoterapéutica (Baum, 1994; Skinner, 1953, 1957, 1969 y 1974), por lo que se muestra como una alternativa viable ante las demandas de las personas consultantes. Asimismo, no cuenta con particularidades específicas en cuanto a la "duración del tratamiento", por lo que se puede poner en marcha en los intervalos de tiempo que se consideren más apropiados para las características de los individuos que asisten a la consulta psicológica.

Igualmente, es importante resaltar la importancia de la flexibilidad de la АCT en una sociedad como la actual, donde cada vez con más frecuencia se deben poner en práctica estrategias innovadoras que permitan a las personas recibir el tratamiento más adecuado para sus problemáticas, dentro de las múltiples limitaciones de una vida contemporánea. Esta particularidad de la ACT permite que se implemente, mediante estrategias diversas como las sesiones presenciales, las sesiones grupales, las sesiones telefónicas, las sesiones mediante el uso de medio digitales, entre otras, lo que permite la oferta de una herramienta psicoterapéutica efectiva $y$ adaptable a las particularidades de cada persona consultante.

La capacidad de adaptarse a multiplicidad de problemáticas y aún así brindar resultados positivos, garantiza además la aplicación de un acercamiento psicológico comprometido con el ejercicio ético de la profesión, dentro de un contexto donde los terapeutas deben contar con la responsabilidad de instrumentalizarse 
con procedimientos que justamente garanticen un pronóstico positivo para una variedad de problemáticas.

Esta diferencia viene dada por la característica de la АCT, la cual consiste en partir de las vivencias de las personas consultantes como insumo orientador del proceso de intervención psicoterapéutica, partiendo entonces de un modelo completamente ideográfico de tratamiento psicológico. Es importante rescatar que, aunque la mayoría de orientaciones de tratamiento clínico parten de las vivencias en específico de los sujetos, en este tipo de terapia se hace énfasis en que son estas experiencias las que van a dirigir el proceso de intervención, por lo que no solo se convierten en insumo para el planeamiento estratégico de las sesiones por parte del terapeuta, sino que se involucra activamente a la persona en su propio proceso de cambio y de búsqueda de su bienestar.

Finalmente, es importante considerar que la sociedad contemporánea presenta una serie de demandas en la vida de las personas, la cual en muchas ocasiones impide el desarrollo $y$ la perpetuación de un modo de vida saludable, por tanto, las aproximaciones clínicas que permitan aminorar el malestar psicológico de las personas de manera efectiva $y$ eficiente deben ser consideradas como una responsabilidad, que todo individuo comprometido con la salud mental de la población debe adoptar dentro de su práctica profesional.

\section{REFERENCIAS BIBLIOGRÁFICAS}

Arch, J. y Craske, M. "Acceptance and Commitment Therapy and Cognitive Behavioral Therapy for anxiety disorders: different treatments, similar mechanisms?". Clinical Psychology: Science and Practice 15. 2008: 263-279.

Baum, W. Understanding behaviorism. Science, behavior, and culture. New York: HarperCollins College Publishers, 1994.

Berman, M.; Boutelle, K. y Crow, S. "A case series investigating Acceptance and Commitment Therapy as a treatment for previously treated, unremitted patients with anorexia nervosa”. European Eating Disorders Review 17 (6). 2009: 426-434.
Blackledge, J. y Hayes, S. "Emotion regulation in Acceptance and Commitment Therapy". Journal of Clinical Psychology 57. 2001: 243-255.

Bricker, J.; Mann, S.; Marek, P.; Liu, J. y Peterson, A. "Telephone-delivered Acceptance and Commitment Therapy for adult smoking cessation: a feasibility study". Nicotine \& Tobacco Research 12 (4). 2010: 454-458.

Carrascoso, F. y Valdivia, S. "Acceptance and Commitment Therapy (ACT) in the treatment of panic disorder: some considerations from the research on basic processes". International Journal of Psychology and Psychological Therapy 9 (3). 2009: 299-315.

Dougher, M. "The act of acceptance". Acceptance and Change: Content and Context in Psychotherapy. Hayes, S.; Jacobson, N.; Follette, V. y Dougher, M. (eds.). Reno: Context Press, 1994.

Gaudiano, B. "Evaluating Acceptance and Commitment Therapy: an analysis of a recent critique". International Journal of Behavioral Consultation and Therapy 5 (3-4). 2009: 311-329.

Hayes, S. "Content, context and the types of psychological acceptance". Acceptance and Change: Content and Context in Psychotherapy. Hayes, S.; Jacobson, N.; Follette, V. y Dougher, M. (eds.). Reno: Context Press, 1994.

Hayes, S. "Climbing our hills: a beginning conversation on the comparison of Acceptance and Commitment Therapy and Traditional Cognitive Behavioral Therapy". Clinical Psychology: Science and Practice 15. 2008: 286-295.

Hayes, S.; Barnes-Homes, D. y Roche, B. Relational frame theory. A postskinnerian account of human language and cognition. New York: Kluwer Academic/Plenum Publishers, 2001.

Hernández-López, M.; Luciano, M.; Bricker, J.; Roales-Nieto, J. y Montesinos, F. "Acceptance and Commitment Therapy for smoking cessation: a preliminary study of its effectiveness in comparison 
with Cognitive Behavioral Therapy". Psychology of Addictive Behaviors 23 (4). 2009: 723-730.

Hofmann, S. "Acceptance and Commitment Therapy: new wave or morita therapy?". Clinical Psychology: Science and Practice 15. 2008: 280-285.

Honarparvaran, N.; Tabrizy, M.; Navabinejad, S.; Shafiabady, A. y Moradi, M. "The efficacy of Acceptance and Commitment Therapy (АCT) training with regard to reducing sexual dissatisfaction among couples". European Journal of Social Sciences 15 (1). 2010: 166-172.

Luciano, C.; Páez, M. y Valdivia, S. "La Terapia de Aceptación y Compromiso (ACT) en el consumo de sustancias como estrategia de evitación experiencial". International Journal of Clinical and Health Psychology 10 (1). 2010: 141-165.

Ossman, W.; Wilson, K.; Storaasli, R. y McNeill, J. "A preliminary investigation of the use of Acceptance and Commitment Therapy in group treatment for social phobia". International Journal of Psychology and Psychological Therapy 6 (3). 2006: 397416.

Páez, M.; Gutiérrez, O.; Valdivia, S. y Luciano, C. "Terapia de Aceptación y Compromiso (АСТ) y la importancia de los valores personales en el contexto de la terapia psicológica”. International Journal of Psychology and Psychological Therapy 6 (1). 2006: 1-20.

Petersen, C. y Zettke, R. "Treating in patients with comorbid depression and alcohol use disorders: a comparison of Acceptance and Commitment Therapy versus treatment as usual". The Psychological Record 59. 2009: 521-536.

Rey, C. "La Terapia de Aceptación y Compromiso (AСT): sus aplicaciones $y$ principales fundamentos conceptuales, teóricos y metodológicos". Suma Psicológica 11 (2). 2004: 267-284.

Robb, H. "Values as leading principles in Acceptance and Commitment Therapy". International Journal of Behavioral Consultation and Therapy 3 (1). 2007: 118-122.
Ruiz, F. "A review of Acceptance and Commitment Therapy (ACT) empirical evidence: correlational, experimental psychopathology, component and outcome studies. International Journal of Psychology and Psychological Therapy 10 (1). 2010: 125-162.

Ruiz, F. y Luciano, C. "Eficacia de la Terapia de Aceptación y Compromiso (ACT) en la mejora del rendimiento ajedrecístico de jóvenes promesas". Psicothema 21 (3). 2009: 347-352.

Skinner, B. Science and Human Behavior. New York: The Free Press, 1953.

Skinner, B. Verbal Behavior. New Jersey: Prentice Hall, 1957.

Skinner, B. Contingencies of reinforcement. New York: Appleton-Century-Crofts, 1969.

Skinner, B. About behaviorism. New York: Vintage Books, 1974.

Smout, M.; Longo, M.; Harrison, S.; Minniti, R.; Wickes, W. y White, J. "Psychosocial treatment for methamphetamine use disorders: a preliminary randomized controlled trial of Cognitive Behavior Therapy and Acceptance and Commitment Therapy". Substance Abuse 31. 2010: 98-107.

Strosahl, K. y Robinson, P. The mindfulness and acceptance workbook for depression. Using Acceptance and Commitment Therapy to move through depression and create a life worth living. Oakland: New Harbinger Publications Inc., 2008.

Twohig, M.; Hayes, S.; Plum, J.; Pruitt, L.; Collins, A.; Hazlett-Stevens, H. et ál. "A randomized clinical trial of Acceptance and Commitment Therapy versus Progressive Relaxation Training for obsessive-compulsive disorder". Journal of Consulting and Clinical Psychology 78 (5). 2010: 705-716.

Vilardaga, R.; Hayes, S. y Schelin, L. "Philosophical, theoretical and empirical foundations of Acceptance and Commitment Therapy". Anuario de Psicología 38 (1). 2007: 117-128. 
Watzlawick, P.; Weakland, J. y Fisch, R. Change: principles of problem formation and problem resolution. New York: W. W. Norton \& Company, 1974.

Wilson, K. y Luciano, C. Terapia de Aceptación y Compromiso (ACT): un tratamiento conductual orientado a los valores. Madrid: Ediciones Pirámide, 2002.
Yovel, I. "Acceptance and Commitment Therapy and the new generation of cognitive behavioral treatments". Israel Journal of Psychiatry \& Related Sciences 46 (4). 2009: 304-309.

Fecha de ingreso: 02/05/2012 Fecha de aprobación: 03/07/2012 